\title{
Seletividade de agrotóxicos usados na produção integrada de maçã para adultos de Trichogramma pretiosum
}

\author{
Cristiane Gindri Manzoni(1), Anderson Dionei Grützmacher(1), Fabrizio Pinheiro Giolo(1), \\ Wagner da Roza Härter ${ }^{(1)}$ e Cristiane Müller ${ }^{(1)}$
}

(1)Universidade Federal de Pelotas, Dep. de Fitossanidade, Campus Universitário, Caixa Postal 354, CEP 96010-900 Pelotas, RS. E-mail: cristianemanzoni@hotmail.com, adgrutzm@ufpel.tche.br, fgiolo.faem@ufpel.tche.br, wharter.faem@ufpel.tche.br, crismiler@yahoo.com.br

\begin{abstract}
Resumo - O objetivo deste trabalho foi avaliar a seletividade de 12 agrotóxicos usados na produção integrada de maçã, em laboratório (temperatura $25 \pm 1{ }^{\circ} \mathrm{C}$, umidade relativa $70 \pm 10 \%$ e fotófase de 14 horas), tendo-se exposto adultos de Trichogramma pretiosum a resíduos secos dos agrotóxicos, na máxima dosagem recomendada para uso em campo, e tendo-se posteriormente mensurado o número de ovos parasitados por fêmea. Reduções no parasitismo, em relação à testemunha (água), foram utilizadas para classificar os agrotóxicos em inócuo $(<30 \%)$, levemente nocivo (30-79\%), moderadamente nocivo (80-99\%) e nocivo ( $>99 \%)$. Foram inócuos os acaricidas Envidor (espirodiclofeno), Kendo 50 SC (fenpiroximato) e Ortus 50 SC (fenpiroximato), os fungicidas Antracol 700 PM (propinebe), Midas BR (famoxadona + mancozebe), Palisade (fluquinconazol), Persist SC (mancozebe) e Systhane PM (miclobutanil) e o inseticida Mimic 240 SC (tebufenozida); o herbicida Polaris (glifosato) foi levemente nocivo; os herbicidas Finale (glufosinato sal de amônio) e Roundup Original (glifosato) foram moderadamente nocivos a T. pretiosum.
\end{abstract}

Termos para indexação: parasitóide de ovos, manejo integrado de pragas, pesticidas.

\section{Side effects of pesticides used in integrated production of apple in adults of Trichogramma pretiosum}

\begin{abstract}
The objective of this work was to evaluate the side effects of 12 pesticides used in the integrated production of apple, in laboratory $\left(25 \pm 1^{\circ} \mathrm{C}\right.$ temperature, $70 \pm 10 \%$ relative humidity and 14 hours photophase), exposing adults of Trichogramma pretiosum to fresh dry pesticide film, in the maximum dosage recommended for field use, with subsequent assessement of the number of eggs parasitized per female. The reduction in the parasitism, compared with the control (water), was used to classify the pesticides as harmless $(<30 \%)$, slightly harmful (30-79\%), moderately harmful (80-99\%) and harmful (>99\%). The acaricides Envidor (spirodiclofen), Kendo 50 SC (fenpyroximate) and Ortus 50 SC (fenpyroximate), the fungicides Antracol 700 PM (propineb), Midas BR (famoxadone + mancozeb), Palisade (fluquinconazole), Persist SC (mancozeb) and Systhane PM (myclobutanil) and the insecticide Mimic 240 SC (tebufenozide) were harmless; the herbicide Polaris (glyphosate) was slightly harmful; the herbicides Finale (gluphosinate-ammonium) and Roundup Original (glyphosate) were moderately harmful to $T$. pretiosum.
\end{abstract}

Index terms: eggs parasitoids, integrated pest management, pesticides.

\section{Introdução}

A produção brasileira de maçãs, na safra de 2004, foi de aproximadamente 973 mil toneladas, com rendimento médio de 29,63 tha-1. As principais áreas de cultivo estão nos estados de Santa Catarina e do Rio Grande do Sul, responsáveis por cerca de $96 \%$ da produção nacional (IBGE, 2005). No entanto, a produção é, a cada safra, comprometida pela ação de insetos-praga, com destaque para os tortricídeos Bonagota cranaodes (Meyrick,
1937) e Grapholita molesta (Busck, 1916) (Lepidoptera: Tortricidae) (Kovaleski, 2004).

O manejo para o controle desses insetos é bastante dificultado, em razão de seus hábitos e comportamento. As lagartas de B. cranaodes se abrigam em cartuchos formados por folhas ou no cálice de frutos, enquanto as lagartas de G. molesta alojam-se no interior dos ponteiros ou de frutos. $\mathrm{O}$ controle geralmente é realizado por meio de inúmeras aplicações de inseticidas de amplo espectro, principalmente organofosforados (Botton et al., 2000). 
Além disso, são efetuadas aplicações de outros agrotóxicos, principalmente fungicidas, acaricidas e herbicidas, que podem interferir negativamente sobre os agentes de controle biológico desses insetos-praga.

$\mathrm{Na}$ produção integrada de maçã (PIM) são preconizadas ações que visam a conservar os inimigos naturais das pragas, entre elas o uso de agrotóxicos seletivos. Segundo Kovaleski et al. (2000), é provável que após algumas safras com menor volume de aplicações de agrotóxicos, as áreas de PIM venham a exibir maiores populações de organismos benéficos, e que seja favorecido o controle biológico natural.

Entre os inimigos naturais, destacam-se os parasitóides de ovos da família Trichogrammatidae, em especial do gênero Trichogramma, que apresentam distribuição mundial, associados a diversas espécies de insetos-praga em diferentes cultivos (Pinto, 1997). No sul do Brasil, na década de 80, Lorenzato (1988) mencionou o parasitismo de Trichogramma sp. em ovos da lagartaenroladeira. Mais recentemente, Monteiro et al. (2004) também relataram o parasitismo de Trichogramma pretiosum Riley, 1879 (Hymenoptera: Trichogrammatidae), em posturas do mesmo lepidóptero, em pomares comerciais de macieira. Na América do Norte, Pinto et al. (2002) mencionaram o parasitismo de T. pretiosum em posturas de G. molesta em macieiras.

No Brasil, estudos para determinar os efeitos de agrotóxicos sobre organismos benéficos em macieira foram realizados com predadores como Neoseiulus californicus (McGregor, 1954) (Acari: Phytoseiidae) (Monteiro, 2001; Meyer, 2003) e Chrysoperla externa (Hagen, 1861) (Neuroptera: Chrysopidae) (Ferreira et al., 2005). Trabalhos com parasitóides, especificamente do gênero Trichogramma, para avaliar o efeito de agrotóxicos usados na PIM, não são conhecidos na literatura nacional.

Considerando-se o potencial de utilização de T. pretiosum, associado ao controle químico na macieira, realizou-se este trabalho com o objetivo de avaliar a seletividade de 12 agrotóxicos usados na PIM, para adultos de $T$. pretiosum, em laboratório.

\section{Material e Métodos}

Os experimentos foram executados de acordo com as metodologias laboratoriais padronizadas pelo grupo de trabalho da International Organization for Biological and Integrated Control of Noxious Animals and Plants (IOBC), West Palaearctic Regional Section (WPRS) (Hassan et al., 2000).

O material biológico, utilizado nos experimentos, foi constituído por parasitóides de ovos da espécie T. pretiosum, coletados em posturas de G. molesta, na cultura do pessegueiro, em Pelotas, RS, em 2003. Com esses insetos foi estabelecida uma criação em laboratório, sob condições controladas de temperatura $\left(25 \pm 1^{\circ} \mathrm{C}\right)$, umidade relativa $(70 \pm 10 \%)$ e fotófase de 14 horas, utilizando-se ovos inviabilizados (Stein \& Parra, 1987) do hospedeiro alternativo Anagasta kuehniella (Zeller, 1879) (Lepidoptera: Pyralidae), criado segundo metodologia descrita por Parra (1997).

Em razão da capacidade operacional do sistema instalado no laboratório, os estudos de seletividade foram divididos em três experimentos, tendo sido avaliados os agrotóxicos (dosagens da formulação do produto comercial): Experimento I - Antracol 700 PM (propinebe, $4 \mathrm{~kg} \mathrm{ha}^{-1}$ ), Persist SC (mancozebe,

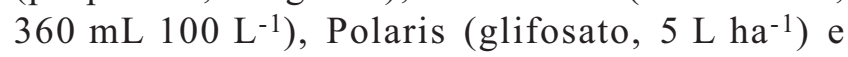

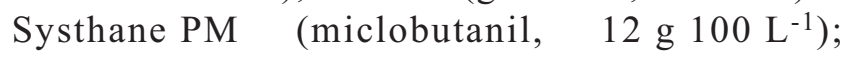
Experimento II - Midas BR (famoxadona + mancozebe,

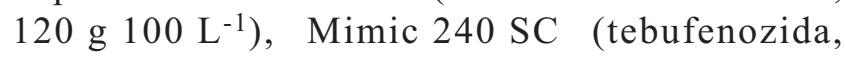

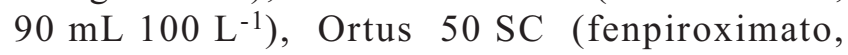

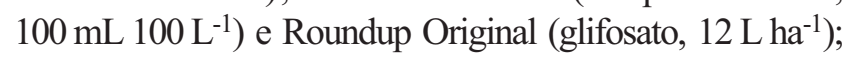

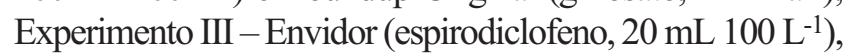
Finale (glufosinato sal de amônio, $2 \mathrm{~L} \mathrm{ha}^{-1}$ ), Kendo $50 \mathrm{SC}$

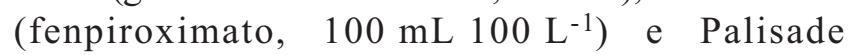
(fluquinconazol, $20 \mathrm{~mL} 100 \mathrm{~L}^{-1}$ ). Esses agrotóxicos estão recomendados na PIM e foram testados na máxima dosagem de registro para a cultura (Tabela 1). O inseticida Dipterex 500, na dosagem de $300 \mathrm{~mL}$ da formulação comercial por $100 \mathrm{~L}$, foi utilizado como padrão de toxicidade em todos os experimentos, por ser reconhecidamente nocivo a parasitóides do gênero Trichogramma (Grützmacher et al., 2004), e a testemunha foi constituída por água.

Os testes de seletividade foram realizados em laboratório (temperatura $25 \pm 1^{\circ} \mathrm{C}$, umidade relativa $70 \pm 10 \%$ e fotófase de 14 horas), tendo-se exposto adultos de T. pretiosum, estágio de vida mais sensível (Hassan et al., 2000), a resíduos secos dos agrotóxicos. As formulações foram aplicadas diretamente sobre placas de vidro de $2 \mathrm{~mm}(13 \times 13 \mathrm{~cm})$, por meio de pulverizadores manuais $(580 \mathrm{~mL})$ calibrados para depositar o volume de $1,75 \pm 0,25 \mathrm{mg}$ de calda por $\mathrm{cm}^{2}$ de superfície, aferido por balança eletrônica de precisão. Depois de tratadas, as placas permaneceram à 
temperatura ambiente por três horas para secagem da calda aplicada que formou uma película seca. Gaiolas de exposição foram confeccionadas com duas placas tratadas, fixadas por presilhas a uma moldura retangular de alumínio (13 cm de comprimento x 1,5 $\mathrm{cm}$ de altura x $1 \mathrm{~cm}$ de largura). Em três dos lados da moldura de alumínio havia orifícios para ventilação (diâmetro aproximado de $1 \mathrm{~cm}$ ). O quarto lado da moldura possuía dois orifícios: um $(3,5 \times 1 \mathrm{~cm})$ para introdução dos ovos do hospedeiro A. kuehniella, a serem parasitados, e alimento composto por $3 \mathrm{~g}$ de gelatina, $100 \mathrm{~mL}$ de água e $200 \mathrm{~g}$ de mel, para os insetos em teste; e o outro (diâmetro de $1 \mathrm{~cm}$ ) para inserção dos parasitóides, através da conexão dos tubos de emergência.

Cada tubo de emergência (ampola de vidro transparente com $120 \mathrm{~mm}$ de comprimento x $20 \mathrm{~mm}$ de diâmetro em uma das extremidades e $7 \mathrm{~mm}$ na outra) foi constituído por um círculo ( $1 \mathrm{~cm}$ de diâmetro), com $250 \pm 50$ ovos previamente parasitados, aderido a uma tira de papel (80x15 mm) com alimento. Esses tubos com adultos de T. pretiosum, com aproximadamente 24 horas de idade, foram conectados às gaiolas de exposição seis horas após a pulverização dos produtos, para permitir a entrada dos parasitóides no interior das gaiolas. Transcorridas 16 horas, os tubos foram desconectados e mantidos sob as mesmas condições do experimento por mais três dias, para que ocorresse a total emergência dos adultos, o que permitiu o cálculo da população inicial do parasitóide. Para se evitar o aumento na concentração de gases tóxicos no interior das gaiolas de exposição, foi acoplado a elas um sistema de sucção de ar (Hassan et al., 2000).

Seis horas após a desconexão dos tubos de emergência, foram oferecidos os primeiros cartões com ovos de A. kuehniella (cada cartão com três círculos de $1 \mathrm{~cm}$ de diâmetro, com $450 \pm 50$ ovos) e alimento. Os cartões foram oferecidos em sobreposição, para serem parasitados a partir de 24 (três cartões), 48 (dois cartões) e 96 horas (um cartão) após a pulverização, num período total aproximado de 144 horas, com disponibilidade de ovos para o parasitismo. Sete dias após a pulverização, as gaiolas foram desmontadas. Os cartões oferecidos ao parasitismo foram armazenados nas mesmas condições do teste por mais três dias, período suficiente para que houvesse o escurecimento de todos os ovos parasitados.

Para determinar o número de fêmeas, em cada gaiola, foram contados os ovos parasitados em cada círculo utilizado na confecção dos tubos de emergência. Este valor foi multiplicado pelo número de parasitóides por ovo da população do inseto. O valor obtido foi subtraído do número de adultos que permaneceram no interior do tubo de emergência e multiplicado pela razão sexual da população. Os parâmetros número de parasitóides por ovo e razão sexual foram obtidos mediante avaliação de quatro círculos de $1 \mathrm{~cm}$ de diâmetro com ovos parasitados, tendo sido esses círculos retirados do mesmo cartão utilizado na confecção dos tubos de emergência.

O número médio de ovos parasitados por fêmea, em cada tratamento, foi utilizado para calcular o parasitismo. A redução no parasitismo, para cada agrotóxico, foi determinada por meio da comparação com a testemunha (água) e corrigida pela fórmula de Abbott (1925). Com base nessas porcentagens de redução no parasitismo, os agrotóxicos testados foram classificados segundo a IOBC/WPRS em: 1$)$ inócuo $(<30 \%)$; 2) levemente nocivo (30-79\%); 3) moderadamente nocivo (80-99\%); e 4) nocivo (>99\%). A classificação dos

Tabela 1. Agrotóxicos avaliados nos testes de seletividade para adultos de Trichogramma pretiosum, com a máxima dosagem do produto formulado recomendado na produção integrada de maçã.

\begin{tabular}{|c|c|c|c|c|c|c|}
\hline Nome técnico & Formulação comercial & Classe & Grupo químico & Dosagem & $\mathrm{Cia}^{(1)}$ & $\mathrm{Cfc}^{(2)}$ \\
\hline Espirodiclofeno & Envidor & Acaricida & Cetoenol & $20^{(3)}$ & 0,005 & 0,020 \\
\hline Famoxadona + Mancozebe & Midas BR & Fungicida & Oxazolidinadiona + Ditiocarbamato & $120^{(3)}$ & $0,008+0,075$ & 0,120 \\
\hline Fenpiroximato & Kendo $50 \mathrm{SC}$ & Acaricida & Pirazol & $100^{(3)}$ & 0,005 & 0,100 \\
\hline Fenpiroximato & Ortus $50 \mathrm{SC}$ & Acaricida & Pirazol & $100^{(3)}$ & 0,005 & 0,100 \\
\hline Fluquinconazol & Palisade & Fungicida & Triazol & $20^{(3)}$ & 0,005 & 0,020 \\
\hline Glifosato & Polaris & Herbicida & Glicina substituída & $5^{(4)}$ & 0,900 & 2,500 \\
\hline Glifosato & Roundup Original & Herbicida & Glicina substituída & $12^{(4)}$ & 2,160 & 6,000 \\
\hline Glufosinato sal de amônio & Finale & Herbicida & Homoalanina substituída & $2^{(4)}$ & 0,200 & 1,000 \\
\hline Mancozebe & Persist SC & Fungicida & Ditiocarbamato & $360^{(3)}$ & 0,160 & 0,360 \\
\hline Miclobutanil & Systhane PM & Fungicida & Triazol & $12^{(3)}$ & 0,005 & 0,012 \\
\hline Propinebe & Antracol 700 PM & Fungicida & Ditiocarbamato & $4^{(4)}$ & 0,187 & 0,267 \\
\hline Tebufenozida & Mimic $240 \mathrm{SC}$ & Inseticida & Benzohiadrazina & $90^{(3)}$ & 0,022 & 0,090 \\
\hline
\end{tabular}

${ }^{(1)}$ Concentração (\%) testada do ingrediente ativo na calda. ${ }^{(2)}$ Concentração (\%) testada da formulação comercial na calda. ${ }^{(3)}$ Dosagem da formulação comercial em grama ou mililitro por $100 \mathrm{~L} .{ }^{(4)}$ Dosagem da formulação comercial em litro ou quilograma por hectare. 
agrotóxicos foi realizada com base no produto comercial, uma vez que um mesmo ingrediente ativo pode estar em distintas formulações comerciais e pode ocasionar impacto diferenciado sobre o inseto-teste (Hassan et al., 2000).

Cada gaiola de contato foi considerada uma unidade experimental. Os experimentos foram inteiramente casualizados e delineados com quatro repetições por tratamento. Os dados obtidos, referentes ao número de ovos parasitados por fêmea, foram submetidos à análise de variação, e as médias dos tratamentos foram comparadas pelo teste de Tukey $(\mathrm{p} \leq 0,05)$.

\section{Resultados e Discussão}

O número médio de fêmeas do parasitóide T. pretiosum, no interior das gaiolas de exposição, ficou entre 82,75 e 128,27 fêmeas por gaiola no Experimento I, 152,40 e 241,82 fêmeas por gaiola no Experimento II e entre 107,45 e 140,45 fêmeas por gaiola no Experimento III (Tabela 2). Estes resultados estão de acordo com a metodologia da IOBC, que recomenda um número entre 50 e 400 parasitóides por gaiola de contato, para testes de seletividade em laboratório, com Trichogramma cacoeciae Marchal, 1927
(Hymenoptera: Trichogrammatidae) (Hassan, 1998a; Hassan et al., 2000), para uma adequada mensuração dos resultados, que são expressos em número de ovos parasitados por fêmea.

O número médio de ovos parasitados, por fêmea de T. pretiosum, apresentou variação significativa entre os agrotóxicos testados (Tabela 2). Na testemunha (água), os valores obtidos foram de 31 ovos parasitados por fêmea no Experimento I, 28,10 ovos parasitados por fêmea no Experimento II, e 42,43 ovos parasitados por fêmea no Experimento III. Este é um parâmetro importante, que valida os testes de seletividade com parasitóides de ovos, executados conforme a metodologia da IOBC/WPRS, visto que a classificação dos agrotóxicos baseia-se na redução do parasitismo em relação à testemunha. Para a espécie T. cacoeciae, está preconizado o mínimo de 15 ovos parasitados por fêmea (Hassan et al., 2000). Porém, para testes com T. pretiosum, esse limite mínimo ainda não está estabelecido.

Rocha \& Carvalho (2004), em seu trabalho que visava à adaptação da metodologia da IOBC, para condições do Brasil, obtiveram um número médio de 12,90 ovos parasitados por fêmea de $T$. pretiosum na testemunha, portanto inferior aos verificados neste

Tabela 2. Efeito de agrotóxicos usados na produção integrada de maçã, sobre o número médio $( \pm$ EP) de ovos parasitados por fêmea, redução (\%) na capacidade de parasitismo de T. pretiosum, e classificação de seletividade destes produtos. Temperatura $25 \pm 1^{\circ} \mathrm{C}$; UR: $70 \pm 10 \%$; fotófase: 14 horas.

\begin{tabular}{|c|c|c|c|c|c|}
\hline Formulação comercial & Dosagem & Número de fêmeas por gaiola & Ovos parasitados por fêmea ${ }^{(1)}$ & $\mathrm{RP}^{(2)}$ & Classes $^{(3)}$ \\
\hline & & \multicolumn{4}{|c|}{ Experimento I } \\
\hline Testemunha & - & $128,27 \pm 7,93$ & $31,00 \pm 1,14 \mathrm{~b}$ & - & - \\
\hline Antracol 700 PM & $4^{(4)}$ & $104,21 \pm 5,57$ & $29,00 \pm 1,90 b$ & 6,45 & 1 \\
\hline Persist SC & $360^{(5)}$ & $86,56 \pm 2,55$ & $41,84 \pm 2,21 \mathrm{a}$ & 0,00 & 1 \\
\hline Systhane PM & $12^{(5)}$ & $98,44 \pm 12,75$ & $31,95 \pm 3,65 b$ & 0,00 & 1 \\
\hline Polaris & $5^{(4)}$ & $100,74 \pm 1,46$ & $10,35 \pm 1,97 \mathrm{c}$ & 66,61 & 2 \\
\hline Dipterex 500 (Padrão) & $300^{(5)}$ & $82,75 \pm 9,01$ & $0,00 \pm 0,00 \mathrm{~d}$ & 100,00 & 4 \\
\hline \multicolumn{6}{|c|}{ Experimento II } \\
\hline Testemunha & - & $241,82 \pm 32,68$ & $28,10 \pm 3,95 \mathrm{a}$ & - & - \\
\hline Midas BR & $120^{(5)}$ & $174,45 \pm 17,48$ & $26,14 \pm 3,30 \mathrm{a}$ & 6,98 & 1 \\
\hline Mimic $240 \mathrm{SC}$ & $90^{(5)}$ & $182,65 \pm 15,53$ & $24,79 \pm 4,41 \mathrm{a}$ & 11,78 & 1 \\
\hline Ortus $50 \mathrm{SC}$ & $100^{(5)}$ & $152,40 \pm 21,22$ & $32,76 \pm 3,41 \mathrm{a}$ & 0,00 & 1 \\
\hline Roundup Original & $12^{(4)}$ & $180,91 \pm 12,72$ & $1,01 \pm 0,19 \mathrm{~b}$ & 96,41 & 3 \\
\hline Dipterex 500 (Padrão) & $300^{(5)}$ & $182,33 \pm 17,38$ & $0,00 \pm 0,00 \mathrm{~b}$ & 100,00 & 4 \\
\hline \multicolumn{6}{|c|}{ Experimento III } \\
\hline Testemunha & - & $127,19 \pm 2,64$ & $42,43 \pm 1,59 \mathrm{ab}$ & - & - \\
\hline Envidor & $20^{(5)}$ & $107,45 \pm 1,56$ & $43,58 \pm 1,87 \mathrm{a}$ & 0,00 & 1 \\
\hline Kendo $50 \mathrm{SC}$ & $100^{(5)}$ & $115,80 \pm 4,68$ & $33,49 \pm 2,52 b$ & 21,07 & 1 \\
\hline Palisade & $20^{(5)}$ & $138,45 \pm 6,03$ & $34,02 \pm 4,14 \mathrm{ab}$ & 19,82 & 1 \\
\hline Finale & $2^{(4)}$ & $130,89 \pm 10,21$ & $1,87 \pm 0,43 \mathrm{c}$ & 95,59 & 3 \\
\hline Dipterex 500 (Padrão) & $300^{(5)}$ & $140,45 \pm 9,41$ & $0,00 \pm 0,00 \mathrm{c}$ & 100,00 & 4 \\
\hline
\end{tabular}

${ }^{(1)}$ Médias seguidas por letras iguais não diferem entre si pelo teste de Tukey $(\mathrm{p} \leq 0,05)$. (2) Redução no parasitismo comparado com a testemunha. ${ }^{(3)}$ Classes da IOBC/WPRS para teste de seletividade sobre Trichogramma: $1=$ inócuo $(<30 \%), 2=$ levemente nocivo $(30-79 \%), 3=$ moderadamente nocivo $(80-99 \%), 4$ = nocivo $(>99 \%)$. ${ }^{(4)}$ Dosagem da formulação comercial em litro ou quilograma por hectare. ${ }^{(5)}$ Dosagem da formulação comercial em grama ou mililitro por $100 \mathrm{~L}$. 
trabalho. Esse baixo valor, provavelmente, se deve a um número reduzido de ovos do hospedeiro por fêmea, o que promoveu competição intraespecífica. Estudos realizados por Maceda et al. (2003) demonstraram que fêmeas de T. pretiosum, à temperatura de $25^{\circ} \mathrm{C}$, parasitaram uma média de 5,74 ovos por dia. Esses valores são bastante próximos aos resultados obtidos neste trabalho, se considerarmos que ovos do hospedeiro A. kuehniella foram oferecidos para o parasitismo, durante o período aproximado de seis dias (cerca de 144 horas).

As formulações comerciais de agrotóxicos testadas afetaram distintamente o parasitismo de fêmeas de T. pretiosum, e os herbicidas foram mais nocivos em relação aos demais produtos avaliados (Tabela 2). O inseticida Dipterex 500 (triclorfom), empregado por pomicultores no controle de grafolita e utilizado como padrão de toxicidade, foi enquadrado na classe 4 (nocivo) nos três experimentos (Tabela 2), com 100\% de redução no parasitismo, conforme mencionado por Grützmacher et al. (2004).

Os acaricidas Envidor (espirodiclofeno), Kendo 50 SC (fenpiroximato) e Ortus $50 \mathrm{SC}$ (fenpiroximato), nas concentrações testadas, foram inócuos (classe 1) a fêmeas de T. pretiosum (Tabela 2). Entretanto, Kendo 50 SC acarretou uma redução de $21,07 \%$ no parasitismo das fêmeas. Ortus $50 \mathrm{SC}$, apesar de ser formulado com o mesmo ingrediente ativo (i.a.), fenpiroximato, não interferiu no parasitismo, e demonstrou ser imprescindível mencionar a formulação comercial na publicação dos resultados referentes a testes de seletividade.

Nas concentrações testadas, os fungicidas Antracol 700 PM (propinebe), Midas BR (famoxadone + mancozebe), Palisade (fluquinconazol), Persist SC (mancozebe) e Systhane PM (miclobutanil) foram considerados inócuos (classe 1), tendo acarretado reduções no parasitismo de fêmeas de T. pretiosum de até $19,82 \%$ (Tabela 2). Porém, um estudo de Hassan (1998b) demonstrou que Antracol 700 PM, na concentração de $0,200 \%$ i.a. (superior à testada neste trabalho), foi levemente nocivo (classe 2) à espécie T. cacoeciae. Resultados similares foram observados por Giolo et al. (2005a), que classificaram o fungicida Persist SC como inócuo a T. pretiosum, na mesma concentração testada neste trabalho. Hassan (1998b) ao testar Dithane Ultra (mancozebe), nas concentrações de $0,100 \%$ e $0,200 \%$ de i.a., sobre T. cacoeciae, atribuíram respectivamente, as classificações levemente nocivo (classe 2) e moderadamente nocivo (classe 3) para o produto. Para os fungicidas Midas BR, Palisade e Sythane PM não existem relatos de testes de seletividade na literatura, sendo os deste trabalho os primeiros resultados gerados para o parasitóide T. pretiosum.

O inseticida regulador de crescimento Mimic 240 SC (tebufenozida) causou redução de $11,78 \%$ no parasitismo de fêmeas de T. pretiosum e foi enquadrado na classe 1 (inócuo) (Tabela 2). Resultados semelhantes foram encontrados por Carvalho et al. (2001), para tebufenozida, ao testar duas linhagens de T. pretiosum em dois experimentos, sendo que em um não houve interferência no parasitismo e em outro obtiveram 10,77\% de redução, porcentual muito próximo ao obtido neste trabalho (Tabela 2). Trabalhos realizados por Cônsoli et al. (1998), que testaram tebufenozida em estágios imaturos (ovo-larva, pré-pupa e pupa) de T. pretiosum, mostraram que esse produto possui efeitos subletais, que acarretam reduções no tempo de desenvolvimento do parasitóide, especialmente quando aplicado durante o estágio de pupa do parasitóide.

Os herbicidas Polaris, na dosagem de $5 \mathrm{~L} \mathrm{ha}^{-1}$, e Roundup Original, na dosagem de $12 \mathrm{~L} \mathrm{ha}^{-1}$, ambos formulados com glifosato, foram considerados, respectivamente, levemente nocivo (classe 2) e moderadamente nocivo (classe 3 ) a fêmeas de $T$. pretiosum (Tabela 2 e 3 ). As distintas classes, observadas neste trabalho para os herbicidas, não devem ser atribuídas à sua formulação comercial, visto que ambos apresentam ligado ao ácido glifosato o mesmo tipo de sal (isopropilamina). A redução na seletividade dos herbicidas pode ser conseqüência, neste estudo, do aumento da concentração de i.a. na calda de aplicação.

Giolo et al. (2005b), ao testar oito formulações comerciais de glifosato sobre $T$. pretiosum, verificaram reduções no parasitismo de 59,89 a 88,19\%; o herbicida Polaris, na dosagem de $8 \mathrm{~L} \mathrm{ha}^{-1}$, superior à avaliada neste trabalho, causou redução de $86,40 \%$ no parasitismo do inseto e foi classificado como moderadamente nocivo (classe 3), e o herbicida Roundup Original, também avaliado na dosagem de $8 \mathrm{~L} \mathrm{ha}^{-1}$, foi considerado moderadamente nocivo (classe 3 ), tendo reduzido em $86,60 \%$ o parasitismo de fêmeas.

O herbicida Finale (glufosinato sal de amônio), na dosagem de $2 \mathrm{~L} \mathrm{ha}^{-1}$ e concentração de $0,200 \%$ de i.a., causou redução de $95,59 \%$ no parasitismo de fêmeas e foi classificado como moderadamente nocivo (classe 3) (Tabela 2). Hassan et al. (1991) testaram o herbicida Basta (glufosinato sal de amônio), na concentração de 
$0,100 \%$ de i.a., e consideraram esse produto levemente nocivo (classe 2) a adultos de T. cacoeciae.

Os testes de toxicidade em laboratório sujeitam os insetos-teste à máxima exposição aos resíduos das formulações comerciais dos agrotóxicos e constituem a primeira etapa da seqüência de testes preconizada pela IOBC/WPRS (Hassan et al., 2000). Conforme a IOBC/WPRS, os acaricidas Envidor, Kendo $50 \mathrm{SC}$ e Ortus $50 \mathrm{SC}$, os fungicidas Antracol $700 \mathrm{PM}$, Midas BR, Palisade, Persist SC e Systhane PM e o inseticida Mimic $240 \mathrm{SC}$, enquadrados na classe 1 (inócuos), e o herbicida Polaris na classe 2 (levemente nocivo), não necessitam ser testados nas etapas subseqüentes (testes com estágios imaturos, de persistência biológica do produto e de campo). No entanto, os herbicidas Finale e Roundup Original, classificados na classe 3 (moderadamente nocivo), precisam ser avaliados em pomares de macieiras, de modo a fornecer resultados definitivos referentes à seletividade desses produtos para T. pretiosum.

\section{Conclusões}

1. Os acaricidas Envidor, Kendo $50 \mathrm{SC}$ e Ortus $50 \mathrm{SC}$; os fungicidas Antracol 700 PM, Midas BR, Palisade, Persist SC e Systhane PM e o inseticida Mimic 240 SC são inócuos (classe 1) a adultos do parasitóide Trichogramma pretiosum.

2. O herbicida Polaris é levemente nocivo (classe 2) a adultos de T. pretiosum.

3. Os herbicidas Finale e Roundup Original são moderadamente nocivos (classe 3 ) a adultos de T. pretiosum.

\section{Agradecimentos}

Ao CNPq e à Fapergs, pelo apoio financeiro para a realização desta pesquisa.

\section{Referências}

ABBOTT, W.S. A method of computing the effectiveness of an insecticide. Journal of Economic Entomology, v.18, p.265-267, 1925.

BOTTON, M.; NAKANO, O.; KOVALESKI, A. Exigências térmicas e estimativa do número de gerações de Bonagota cranaodes (Meyrick) (Lepidoptera : Tortricidae) em regiões produtoras de maçã do Sul do Brasil. Anais da Sociedade Entomológica do Brasil, v.29, p.633$637,2000$.

CARVALHO, G.A. de; PARRA, J.R.P.; BAPTISTA, G.C. de. Impacto de produtos fitossanitários utilizados na cultura do tomateiro na fase adulta de duas linhagens de Trichogramma pretiosum Riley,
1879 (Hymenoptera: Trichogrammatidae). Ciência e Agrotecnologia, v.25, p.560-568, 2001.

CÔNSOLI, F.L.; PARRA, J.R.P.; HASSAN, S.A. Side-effects of insecticides used in tomato fields on the egg parasitoid Trichogramma pretiosum Riley (Hym., Trichogrammatidae), a natural enemy of Tuta absoluta (Meyrick) (Lep., Gelechiidae). Journal of Applied Entomology, v.122, p.43-47, 1998.

FERREIRA, A.J.; CARVALHO, G.A.; BOTTON, M.; MENDONÇA, L.A.; CORREAA, A.R.B. Seletividade de inseticidas usados na cultura da macieira a ovos de Chrysoperla externa (Hagen, 1861) (Neuroptera: Chrysopidae). Ciência Rural, v.35, p.756-762, 2005.

GIOLO, F.P.; GRÜTZMACHER, A.D.; MANZONI, C.G.; FACHINELLO, J.C.; NÖRNBERG, S.D.; STEFANELLO JÚNIOR, G.J. Seletividade de agrotóxicos indicados na produção integrada de pêssego a Trichogramma pretiosum Riley, 1879 (Hymenoptera: Trichogrammatidae). Revista Brasileira de Fruticultura, v.27, p.222-225, 2005a.

GIOLO, F.P.; GRÜTZMACHER, A.D.; PROCÓPIO, S.O.; MANZONI, C.M.; LIMA, C.A.B.; NÖRNBERG, S.D. Seletividade de formulações de glyphosate a Trichogramma pretiosum (Hymenoptera: Trichogrammatidae). Planta Daninha, v.23, p.457$462,2005 b$.

GRÜTZMACHER, A.D.; ZIMMERMANN, O.; YOUSEF, A.; HASSAN, S.A. The side-effects of pesticides used in integrated production of peaches in Brazil on the egg parasitoid Trichogramma cacoeciae Marchal (Hym., Trichogrammatidae). Journal of Applied Entomology, v.128, p.377-383, 2004.

HASSAN, S.A. Guidelines for the evaluation of side effects of plant protection products on Trichogramma cacoeciae Marchal (Hym., Trichogrammatidae). IOBC/WPRS Bulletin, v.21, p.119-128, 1998a.

HASSAN, S.A. The side effects of 161 pesticides on the egg parasitoid Trichogramma cacoeciae Marchal (Hymenoptera, Trichogrammatidae). Egg Parasitoid News, v.10, p.63-76, 1998b.

HASSAN, S.A.; BIGLER, F.; BOGENSCHÜTZ, H.; BOLLER, E.; BRUN, J.; CALIS, J.N.M.; CHIVERTON, P.; COREMANSPELSENEER, J.; DUSO, C.; LEWIS, G.B.; MANSOUR, F.; MORETH, L.; OOMEN, P.A.; OVERMEER, W.P.J.; POLGAR, L.; RIECKMANN, W.; SAMSOE-PETERSEN, L.; STÄUBLI, A.; STERK, G.; TAVARES, K.; TUSET, J.J.; VIGGIANI, G. Results of the fifth joint pesticide testing programme carried out by the IOBC/ WPRS - working group "Pesticides and Beneficial Organisms". Entomophaga, v.36, p.55-67, 1991.

HASSAN, S.A.; HALSALL, N.; GRAY, A.P.; KUEHNER, C.; MOLL, M.; BAKKER, F.M.; ROEMBKE, J.; YOUSEF, A.; NASR, F.; ABDELGADER, H.A. A laboratory method to evaluate the side effects of plant protection products on Trichogramma cacoeciae Marchal (Hym., Trichogrammatidae). In: CANDOLFI, M.P.; BLÜMEL, S.; FORSTER, R.; BAKKER, F.M.; GRIMM, C.; HASSAN, S.A.; HEIMBACH, U.; MEAD-BRIGGS, M.A.; REBER, B.; SCHMUCK, R.; VOGT, H. (Ed.). Guidelines to evaluate side-effects of plant protection products to non-target arthropods. Reinheim: IOBC/WPRS, 2000. p.107-119. 
IBGE. Pesquisa mensal de previsão e acompanhamento das safras agrícolas no ano civil: janeiro 2005. Levantamento Sistemático da Produção Agrícola, v.17, p.1-62, 2005. Disponível em <ftp:// ftp.ibge.gov.br/Producao_Agricola>. Acesso em: 20 ago. 2005.

KOVAlESKI, A. Pragas. In: KOVALESKI, A. (Ed.). Maçã: fitossanidade. Bento Gonçalves: Embrapa Uva e Vinho, 2004. p.1033.

KOVALESKI, A.; SANHUEZA, R.M.V.; RIBEIRO, L.G.; BECHER, W.; BONETI, I.S.; KATSURAYAMA, Y.; PROTAS, J.F. Resultados de pesquisa da produção integrada da maçã (PIM). In: SEMINÁRIO BRASILEIRO DE PRODUÇÃO INTEGRADA DE FRUTAS, 2., 2000, Bento Gonçalves. Anais. Bento Gonçalves: Embrapa Uva e Vinho, 2000. p.87-94. (Embrapa Uva e Vinho. Documentos, 28). Editado por Gilmar Ribeiro Nachtigall e Ana Beatriz Costa Czermainski.

LORENZATO, D. Lepidópteros nocivos em frutíferas rosáceas no Sul do Brasil. Ipagro Informa, v.31, p.71-77, 1988.

MACEDA, A.; HOHMANN, C.L.; SANTOS, H.R. dos. Temperature effects on Trichogramma pretiosum Riley and Trichogrammatoidea annulata de Santis. Brazilian Archives of Biology and Technology, v.46, p.27-32, 2003.

MEYER, G.A. Flutuação populacional de Panonychus ulmi (Koch, 1836) (Acari: Tetranychidae) e seus predadores em pomares de macieira nos sistemas de produção integrada e produção convencional e testes de seletividade em laboratório com Neoseiulus californicus (McGregor, 1954) (Acari: Phytoseiidae). 2003. 91p. Dissertação (Mestrado) - Universidade Federal de Pelotas, Pelotas.

MONTEIRO, L.B. Seletividade de inseticidas a Neoseiulus californicus McGregor (Acari: Phytoseiidae) em macieira no Rio
Grande do Sul. Revista Brasileira de Fruticultura, v.23, p.589$592,2001$.

MONTEIRO, L.B.; SOUZA, A. de; BELLI, E.L.; SILVA, R.B.Q. da; ZUCCHI, R.A. Ocorrência de Trichogramma pretiosum Riley, 1879 (Hymenoptera: Trichogrammatidae) em ovos de Bonagota cranaodes (Meyrick) (Lepidoptera: Tortricidae) em macieira. Revista Brasileira de Fruticultura, v.26, p.171-172, 2004.

PARRA, J.R.P. Técnicas de criação de Anagasta kuehniella, hospedeiro alternativo para produção de Trichogramma. In: PARRA, J.R.P.; ZUCCHI, R.A. (Ed.). Trichogramma e o controle biológico aplicado. Piracicaba: Fealq, 1997. p.121-150.

PINTO, J.D. Taxonomia de Trichogrammatidae (Hymenoptera) com ênfase nos gêneros que parasitam Lepidoptera. In: PARRA, J.R.P.; ZUCCHI, R.A. (Ed.). Trichogramma e o controle biológico aplicado. Piracicaba: Fealq, 1997. p.13-39.

PINTO, J.D.; KOOPMANSCHAP, A.B.; PLATNER, G.R.; STOUTHAMER, R. The North American Trichogramma (Hymenoptera: Trichogrammatidae) parasitizing certain Tortricidae (Lepidoptera) on apple and pear, with ITS2 DNA characterizations and description of a new species. Biological Control, v.23, p.134$142,2002$.

ROCHA, L.C.D.; CARVALHO, G.A. Adaptação da metodologia padrão da IOBC para estudos de seletividade com Trichogramma pretiosum Riley, 1879 (Hymenoptera: Trichogrammatidae) em condições de laboratório. Acta Scientiarium, v.26, p.315-320, 2004.

STEIN, C.P.; PARRA, J.R.P. Uso da radiação ultravioleta para inviabilizar ovos de Anagasta kuehniella (Zeller, 1879) visando estudos com Trichogramma spp. Anais da Sociedade Entomológica do Brasil, v.16, p.229-233, 1987. 